\title{
Validade e reprodutibilidade do Questionário de Hábitos relacionados ao Trabalho (QHT) para trabalhadores do SAMU 192*
}

\section{Validity and reproducibility of the Work-related Habits Questionnaire (HQW) to SAMU 192 workers}

\author{
Fabiana Oliveira Chaise ${ }^{1}$, Ana Paula Kasten ${ }^{1}$, Tássia Silveira Furlanetto ${ }^{1}$, \\ Jorge Pasa ${ }^{1}$, Claudia Tarragô Candotti ${ }^{1}$
}

http://dx.doi.org/10.11606/issn.2238-6149.v27i2p199-215

Chaise FO, Kasten AP, Furlanetto TS, Pasa J, Candotti CT. Validade e reprodutibilidade do Questionário de Hábitos relacionados ao Trabalho (QHT) para trabalhadores do SAMU 192. Rev Ter Ocup Univ São Paulo. 2016 maio-ago.;27(2):199-215.

RESUMO: O estudo teve objetivo de desenvolver um questionário sobre hábitos relacionados ao trabalho (QHT) e verificar sua validade e reprodutibilidade. As etapas do QHT foram: observação de campo e revisão teórica sobre hábitos relacionados ao trabalho de profissionais da área da saúde; descrição e conteúdo; validade de conteúdo e reprodutibilidade. Para análise dos dados foi utilizado estatística descritiva (validade de conteúdo) e percentual de concordância (\%C) e a medida de concordância Kappa (reprodutibilidade). Seis especialistas avaliaram a maior parte do instrumento como excelente, considerando-o válido em seu conteúdo. Os valores de Kappa mostraram concordância moderadas a excelentes entre as respostas ( $\% \mathrm{C}>80 \%$ ), indicando ser o QHT um instrumento reprodutível. Apesar das limitações do QHT tais como ausência de questões sobre etilismo e desequilíbrio entre demanda de trabalho e disponibilidade de recursos humanos, o alcance prático do instrumento está na sua facilidade em obtenção de informações para conhecer o perfil e alguns importantes aspectos de rotina de trabalho dos trabalhadores da saúde, útil para avaliação na área da Terapia Ocupacional.

DESCRITORES: Inquéritos e questionários; Reprodutibilidade dos testes; Estilo de vida; Hábitos; Saúde do trabalhador.
Chaise FO, Kasten AP, Furlanetto TS, Pasa J, Candotti CT. Validity and reproducibility of the Work-related Habits Questionnaire (HQW) to SAMU 192 workers. Rev Ter Ocup Univ São Paulo. 2016 May.-Aug.;27(2):199-215.

\begin{abstract}
The aim of this study was to develop a questionnaire about work-related habits (WHQ) and to verify its validity and reproducibility. The WHQ steps were: field observation and theoretical review about work-related habits of health workers; description and content; content validity, and reproducibility. Descriptive statistics (content validity), percent agreement $(\% \mathrm{C})$, and the measure agreement Kappa (reproducibility) were used for data analysis. Six experts assessed most part of the instrument as excellent, considering its content valid. Kappa values showed moderate to excellent concordance among the answers $(\% \mathrm{C}>80 \%)$ indicating the reproducibility of the WHQ. Despite the limitations of the WHQ, such as absence of questions about alcoholism and imbalance between labor demand and availability of human resources, the practical range of the instrument is in the facility in obtaining information on the profile and on some important aspects of the work routine of health workers, which is useful to evaluate the Occupational Therapy field.
\end{abstract}

KEYWORDS: Surveys and questionnaires; Reproducibility of results; Lifestyle; Habits; Occupational health.

\footnotetext{
*Este trabalho é parte da dissertação de mestrado "Fatores de riscos à saúde cardiovascular e osteomuscular relacionados aos hábitos, estilo de vida e ao trabalho em profissionais do SAMU 192 Porto Alegre/RS" de Fabiana Oliveira Chaise, área Ciências do Movimento Humano pela Universidade Federal do Rio Grande do Sul.

1. Universidade Federal do Rio Grande do Sul.

Endereço para correspondência: Tássia Silveira Furlanetto. Rua Albino Paul, 45 - Bairro Jardim América. São Leopoldo, RS. CEP: 93032-280. E-mail: tassiasf@gmail.com
} 


\section{INTRODUÇÃO}

$\int \begin{aligned} & \text { os últimos anos, são verificadas mudanças } \\ & \text { no mundo do trabalho, com crescente } \\ & \text { estreitamento ou sobreposição da }\end{aligned}$ vida profissional em relação à vida pessoal, em que o trabalhador comprometido com sua instituição se esforça para atingir as demandas solicitadas ${ }^{1}$. Além disso, ocorreram modificações na organização do trabalho nas instituições públicas e privadas, gerando fatores de risco ao adoecimento, tais como: o aumento de horas trabalhadas, o alto grau de simultaneidade de atividades e cobranças, o excesso de metas de trabalho, o processo de tomada de decisão e a pressão excessiva ${ }^{1}$.

Os trabalhadores da área de saúde integram um grupo de risco para o aparecimento de Doenças Osteomusculares relacionadas ao Trabalho (DORT's) e Doenças Cardiovasculares $(\mathrm{DCV})^{2}$. Além disso, a alta prevalência de lombalgia tem sido associada ao risco ergonômico, tais como posturas inadequadas, esforço físico excessivo, carga mecânica e estática osteomuscular, invariabilidade da tarefa.

Esses fatores, associados aos fatores organizacionais, psicossociais e cognitivos ligados ao trabalho são predisponentes ao aparecimento das DORT's ${ }^{3,4}$. Os trabalhadores da saúde apresentam também alta prevalência de hipertensão arterial sistêmica (HAS) e estão expostos aos fatores de risco para o desenvolvimento das DCV, tais como: a obesidade, a dislipidemia, o estresse e o sedentarismo ${ }^{5,6}$.

Neste contexto as transformações do trabalho, além de gerar novas configurações organizacionais, geram repercussões diferenciadas na saúde do trabalhador ${ }^{7}$ e, a consequente necessidade de normatização de medidas de proteção à segurança e à saúde dos trabalhadores. No que se refere aos trabalhadores da área da saúde, foi constituída a Norma Regulamentadora de Segurança e Saúde no Trabalho em Estabelecimentos de Saúde no. 32 de 2005 (NR 32) do Ministério do Trabalho e Emprego - MTE, que institui diretrizes para proteção da saúde dos trabalhadores dos serviços de saúde e daqueles que exercem atividades de promoção e assistência à saúde em geral, bem como estabelece os riscos biológicos, químicos, físicos, entre outros aos quais esses trabalhadores estão expostos. Ela determina que as regras para elaboração e implementação do Programa de Prevenção de Riscos Ambientais (PPRA - NR 9 - de 78 e atualizada em 1994) sejam aplicadas nos serviços de saúde.

A Política Nacional de Saúde do Trabalhador e da Trabalhadora $n^{\circ} 1.823$ de 2012, tem como finalidade definir os princípios, diretrizes e estratégias para o desenvolvimento da atenção integral à saúde do trabalhador. Visa à promoção e a proteção da saúde dos trabalhadores, bem como a redução da morbimortalidade decorrente dos modelos de desenvolvimento e dos processos produtivos.

A condição de saúde, bem como os fatores de risco para o adoecimento dos trabalhadores, podem ser analisados por meio de instrumentos de avaliação. Alguns instrumentos identificam estilo e qualidade de vida, outros mapeiam os fatores de risco ocupacionais e os acidentes de trabalho e outros se restringem a detecção de fatores ou situações de riscos pontuais para o adoecimento, como por exemplo, o aparecimento de doenças de ordem psicológica como o estresse pós-traumático e a Síndrome de Burnout ${ }^{8,9,10}$.

Considerando a necessidade de instrumentos que permitam mapear os fatores de risco para as DORT's e DCV, foi desenvolvido o Questionário de Hábitos relacionados ao Trabalho (QHT). O QHT investiga o trabalho nos aspectos: jornada de trabalho, riscos ocupacionais, estresse no ambiente de trabalho e o aparecimento de DCV e DORT's, e pode ser utilizado tanto na pesquisa, quanto na prática clínica para promover a saúde do trabalhador. Assim, os objetivos do presente artigo são: (1) apresentar o desenvolvimento do QHT, (2) apresentar o resultado do processo de validação de conteúdo do instrumento e (3) avaliar a reprodutibilidade do instrumento.

\section{PROCEDIMENTOS METODOLÓGICOS}

O desenvolvimento do QHT foi realizado em quatro etapas: observação de campo e revisão teórica sobre hábitos de vida no trabalho relacionados aos profissionais que atuam na área da saúde (Etapa 1); descrição de conteúdo do QHT (Etapa 2); validade de conteúdo do QHT (Etapa 3) e avaliação da reprodutibilidade do QHT (Etapa 4). Este estudo foi conduzido nos anos de 2013 e 2014, dentro dos padrões éticos exigidos pela Comissão Nacional de Ética em Pesquisa/Conselho Nacional de Saúde/Ministério da Saúde (CONEP/CNS/MS) e aprovado pelo Comitê de Ética em Pesquisa (CEP) da Universidade Federal do Rio Grande do Sul sob o número de parecer 384.874.

\section{Observação de Campo e Revisão Teórica (Etapa 1)}

A observação de campo foi desenvolvida nos meses de abril e maio de 2013, na sede administrativa do Serviço de Atendimento Móvel de Urgências (SAMU 192) Porto Alegre/RS. Consistiu na convivência diária com os trabalhadores que pertencem às equipes de Suporte 
Básico de Vida (SBV) e Suporte Avançado de Vida (SAV): médicos, enfermeiros, técnicos de enfermagem e condutores de ambulância, e com os trabalhadores pertencentes à central de regulação médica - telefonistas auxiliar de regulação médica (TARM) e setor administrativo. Participamos também dos treinamentos técnicos oferecidos periodicamente pelo Núcleo de Educação em Urgências, o qual é um espaço de formação e capacitação que qualifica o trabalhador na sua atuação no atendimento às urgências.

Durante a observação de campo analisamos o ambiente, a organização, as relações e a jornada de trabalho em todos os setores que compõe o SAMU 192. Nesse período, escutamos informalmente vários relatos dos funcionários, sugerindo que as dores musculoesqueléticas, a HAS e o colesterol alto são manifestações que evidenciam o comprometimento de sua saúde; e que o estresse, o tabagismo e o sedentarismo são alguns fatores de risco à sua saúde. Esses relatos foram anotados, compilados e utilizados para serem agregados ao conteúdo do questionário.

A etapa seguinte foi referente à elaboração dos itens do QHT. Para tanto, procedeu-se uma revisão teórica sobre o tema nas bases de dados PubMed, EBSCO, Embase e Science Direct, no mês de abril de 2013, com a utilização das palavras-chaves: Emergency Medical Technicians [OR] Paramedics, Emergency [AND] Occupational Disease [AND] Risk Factors [AND] Cardiovascular Diseases [AND] Musculoskeletal, Diseases. Os artigos encontrados deveriam preencher os critérios de inclusão: abordar fatores de risco para as DCV e DORT's e envolver trabalhadores do atendimento pré-hospitalar de urgência. Foram excluídos os artigos que: realizavam avaliação de algum tipo de tratamento para esses trabalhadores; abordavam evento único, como por exemplo, as catástrofes; não estavam redigidos na língua inglesa e estudos de caráter qualitativo.

Nessa busca, encontramos alguns estudos utilizando instrumentos para mapear exclusivamente os hábitos de vida, saúde, qualidade de vida, absenteísmo, ambiente e acidentes de trabalho de trabalhadores da saúde ${ }^{9,11-13}$. Entre os achados, observamos: o estresse no ambiente de trabalho (muitas vezes relacionando o estresse pós-traumático como uma possível fonte primária para o adoecimento); as dores musculoesqueléticas; os acidentes de trabalho (principalmente os automobilísticos e com material perfuro-cortante); e os distúrbios do sono.

Com base na observação de campo e na revisão de literatura, desenvolvemos as questões iniciais do QHT, que retratam o universo ocupacional do trabalhador da saúde, englobando ambiente, jornada e estresse relacionado ao trabalho, bem como as doenças ocupacionais e os riscos inerentes às profissões.

\section{Descrição de Conteúdo do QHT (Etapa 2)}

$\mathrm{O}$ QHT é um instrumento de coleta de dados que foi elaborado pelos autores com base na Etapa 1, inicialmente com 30 questões que foram divididas em sete núcleos (Quadro 1). Após a elaboração do QHT, o instrumento passou pela etapa seguinte.

Quadro 1 - Composição de Núcleos do QHT, nº e tipo de questões e seus conteúdos

\begin{tabular}{|c|c|c|c|}
\hline Núcleo & \begin{tabular}{|l|}
$N^{o}$ de \\
Questões
\end{tabular} & \begin{tabular}{|l|} 
Tipo de \\
questões
\end{tabular} & Conteúdo \\
\hline Demográfico & 05 & $\begin{array}{l}\text { Abertas e } \\
\text { fechadas } \\
\text { de múltipla } \\
\text { escolha }\end{array}$ & $\begin{array}{l}\text { Dados pessoais: cor, } \\
\text { sexo, escolaridade, } \\
\text { altura e peso. }\end{array}$ \\
\hline $\begin{array}{l}\text { Ocupacional } \\
\text { (Jornada de } \\
\text { Trabalho) }\end{array}$ & 08 & $\begin{array}{l}\text { Abertas e } \\
\text { fechadas } \\
\text { de múltipla } \\
\text { escolha }\end{array}$ & $\begin{array}{l}\text { Função, carga horária, } \\
\text { regime de trabalho, } \\
\text { horas extras, etc. }\end{array}$ \\
\hline $\begin{array}{l}\text { Ocupacional } \\
\text { (Riscos Físicos, } \\
\text { Químicos, } \\
\text { Biológicos e } \\
\text { Ergonômicos) }\end{array}$ & 02 & $\begin{array}{l}\text { Fechadas } \\
\text { de múltipla } \\
\text { escolha }\end{array}$ & $\begin{array}{l}\text { Contato/exposição } \\
\text { a perfuro-cortantes, } \\
\text { sangue, fluidos, } \\
\text { calor, frio, ruído, etc. } \\
\text { Exigências que a } \\
\text { função inclui: esforço } \\
\text { físico, concentração, } \\
\text { uso do computador, etc. }\end{array}$ \\
\hline $\begin{array}{l}\text { Ocupacional } \\
\text { (Estresse e } \\
\text { Ambiente de } \\
\text { Trabalho) }\end{array}$ & 03 & $\begin{array}{l}\text { Fechadas } \\
\text { de múltipla } \\
\text { escolha }\end{array}$ & $\begin{array}{l}\text { Estresse no ambiente } \\
\text { de trabalho, quanto ao } \\
\text { trabalho em equipe, } \\
\text { apoio da chefia, } \\
\text { restrição de sono, } \\
\text { problemas de saúde } \\
\text { mental. }\end{array}$ \\
\hline $\begin{array}{l}\text { Ocupacional } \\
\text { (Doenças e } \\
\text { Acidentes de } \\
\text { Trabalho) }\end{array}$ & 04 & $\begin{array}{l}\text { Abertas e } \\
\text { fechadas } \\
\text { de múltipla } \\
\text { escolha }\end{array}$ & $\begin{array}{l}\text { Contaminações com } \\
\text { fluidos, acidentes } \\
\text { automobilísticos, } \\
\text { agressões físicas, } \\
\text { absenteísmo, etc. }\end{array}$ \\
\hline Cardiovascular & 05 & $\begin{array}{l}\text { Abertas e } \\
\text { fechadas } \\
\text { de múltipla } \\
\text { escolha }\end{array}$ & $\begin{array}{l}\text { Fatores de riscos } \\
\text { modificáveis e } \\
\text { não modificáveis } \\
\text { e as doenças } \\
\text { cardiovasculares. }\end{array}$ \\
\hline Osteomuscular & 03 & $\begin{array}{l}\text { Abertas e } \\
\text { fechadas } \\
\text { de múltipla } \\
\text { escolha }\end{array}$ & $\begin{array}{l}\text { Diagnóstico de } \\
\text { doença osteomuscular, } \\
\text { frequência e } \\
\text { intensidade da dor e } \\
\text { uso de medicamentos } \\
\text { para dor. }\end{array}$ \\
\hline
\end{tabular}




\section{Validade de Conteúdo do QHT (Etapa 3)}

A validade de conteúdo consiste na apreciação e julgamento do conteúdo do questionário por especialistas com grande experiência profissional e/ou em pesquisa sobre o tema ${ }^{14}$. Foram convidados seis especialistas: dois doutores na área da cardiologia com ênfase em nutrição e fisioterapia, um mestre na área de epidemiologia, um mestre na área da cardiologia com ênfase em urgência, uma doutora em saúde coletiva e uma especialista em fisioterapia nas urgências.

Os especialistas foram convidados via contato telefônico ou por e-mail e todos aceitaram participar do estudo, através da assinatura do termo de consentimento livre e esclarecido, sendo, então, nominados avaliadores do QHT. Os avaliadores participaram das duas fases da Etapa 3: (a) primeira rodada de avaliação e (b) segunda rodada de avaliação. Cada avaliador recebeu uma carta explicando o objetivo do estudo, uma cópia do QHT e o instrumento a ser avaliado. Solicitou-se que o avaliador julgasse cada questão do QHT quanto aos aspectos: (1) Clareza: indica se não existe margem para diferentes interpretações da questão; (2) Aplicabilidade: indica se as questões se aplicam ao propósito do núcleo; (3) Objetividade: indica se a questão é direta e contempla um único aspecto avaliado; (4) Conteúdo: indica se cada questão abrange o conteúdo adequado e se está de acordo com o seu núcleo correspondente. Todos os aspectos deveriam ser julgados pelos avaliadores como excelente, bom ou insuficiente.

No instrumento havia espaço para que os avaliadores acrescentassem sugestões ou observações para cada questão do QHT, podendo indicar também o acréscimo ou a retirada de itens. Com base no parecer dos avaliadores, o QHT foi reformulado e novamente enviado aos avaliadores para que reavaliassem, seguindo os mesmos aspectos da primeira rodada. No momento que os avaliadores não mencionaram mais a necessidade de reformulação, o QHT foi considerado válido quanto ao seu conteúdo, passando para a etapa seguinte. A versão final do QHT está apresentada no Apêndice 1.

\section{Reprodutibilidade do QHT (Etapa 4)}

A etapa da reprodutibilidade foi realizada nos meses de junho a setembro de 2013, pelo método de teste e re-teste, que demonstra a capacidade de uma medida aferir resultados semelhantes em distintas aplicações desde que não haja mudança nas variáveis estudadas ${ }^{15}$. Assim, a versão final do QHT foi aplicado em dois dias distintos pelo mesmo avaliador, com intervalo de tempo de sete dias $^{15}$. O intervalo não foi longo o suficiente para permitir um aprendizado ou mudança de hábitos.

O tamanho da amostra para a etapa da reprodutibilidade foi estimado baseado na prevalência (25\%) dos fatores de risco para DCV e DORT's na população brasileira ${ }^{16}$, com erro de $10 \%$ e nível de significância de 0,05 . Portanto, 43 trabalhadores do SAMU 192 Porto Alegre/RS participaram desta etapa.

\section{Análise Estatística}

Nas questões fechadas, cada uma das possibilidades de resposta foi numerada, sendo as respostas tabuladas. Para as questões abertas, os dados foram compilados em unidades temáticas, as quais também foram numeradas e tabuladas para a análise estatística.

Para a análise estatística foi utilizado o software SPSS versão 17.0. Para análise dos dados da validade de conteúdo foi utilizada estatística descritiva através de tabelas de frequência e porcentagem e para os dados de reprodutibilidade foi utilizado o percentual de concordância $(\% \mathrm{C})$ e a medida de concordância Kappa de Cohen (k). A classificação para concordância entre as respostas pode ser: pobre $(\mathrm{k} \leq 0.2)$, razoável $(0.2<\mathrm{k} \leq 0.4)$, moderada $(0.4<\mathrm{k} \leq 0.6)$, boa $(0.6<\mathrm{k} \leq 0.8)$ ou excelente $(\mathrm{k}<0.8)^{17}$. Somente foram aceitos os valores de $\mathrm{k}$ superiores a 0,4 e os $\%$ C superiores a $80 \%{ }^{18}$.

\section{RESULTADOS}

Os resultados da primeira e da segunda rodada de avaliação da validade de conteúdo do QHT são apresentados na Tabela 1 . As frequências que apresentaram avaliação insuficiente na primeira rodada foram alteradas, seguindo as recomendações dos avaliadores. Os núcleos demográfico e ocupacional foram os que sofreram as maiores alterações na primeira versão do QHT. Na segunda rodada a maioria dos avaliadores conceituou o QHT como excelente em seus aspectos (Tabela 1).

As principais modificações propostas pelos avaliadores na primeira rodada de avaliação foram: alteração no nome de três núcleos, modificações em algumas questões e a inserção de três questões nos núcleos demográfico, ocupacional/jornada de trabalho e ocupacional/estresse, totalizando 33 questões. Na Tabela 2 estão apresentadas as alterações no QHT.

Para os dados de reprodutibilidade participaram 43 trabalhadores que compõe o quadro funcional do SAMU 192 Porto Alegre. A Tabela 3 mostra as características 
socio-demográficas dos participantes, divididos por função.

Os resultados da reprodutibilidade do QHT são apresentados na Tabela 4. Podemos observar que os valores de $\mathrm{k}$ para as questões testadas foram superiores a 0,4 e que os $\% \mathrm{C}$ foram superiores a $80 \%$ com exceção da questão 18 no item jornada de trabalho, o qual foi de $76,7 \%$. O restante das questões do QHT obtiveram valor de $\mathrm{k} 1,0$ e $100 \%$ de concordância, não sendo necessária a modificação de nenhuma questão nesta etapa do estudo.

Tabela 1 - Frequências das avaliações de cada núcleo do QHT entre os especialistas/avaliadores na primeira e segunda rodada de avaliação

\begin{tabular}{|c|c|c|c|c|c|c|c|c|}
\hline \multirow[b]{2}{*}{ Núcleo (questões) } & \multirow[b]{2}{*}{$\begin{array}{c}\text { Avaliadores } \\
(n=6)\end{array}$} & \multirow[b]{2}{*}{ Aspectos } & \multirow[b]{2}{*}{$n *$} & \multicolumn{3}{|c|}{$1^{a}$ Rodada de Avaliação } & \multicolumn{2}{|c|}{$2^{\text {a }}$ Rodada de Avaliação } \\
\hline & & & & $\begin{array}{c}\text { Excelente } \\
\text { n }(\%)\end{array}$ & $\begin{array}{l}\text { Bom } \\
\mathrm{n}(\%)\end{array}$ & $\begin{array}{c}\text { Insuficiente } \\
\mathrm{n}(\%)\end{array}$ & $\begin{array}{c}\text { Excelente } \\
n(\%)\end{array}$ & $\begin{array}{c}\text { Bom } \\
\text { n (\%) }\end{array}$ \\
\hline $\begin{array}{l}\text { Identificação } \\
\text { (1 a 5) }\end{array}$ & 6 & $\begin{array}{l}\text { Clareza, } \\
\text { Aplicabilidade, } \\
\text { Objetividade e } \\
\text { Conteúdo }\end{array}$ & 30 & $25(83,3)$ & $3(10)$ & $2(6,7)$ & $28(93,3)$ & $2(6,7)$ \\
\hline \multirow{4}{*}{$\begin{array}{l}\text { Ocupacional - } \\
\text { Jornada de Trabalho } \\
\text { (6 a 13) }\end{array}$} & \multirow{4}{*}{6} & Clareza & 48 & $35(72,9)$ & $11(22,9)$ & $2(4,2)$ & $46(95,8)$ & $2(4,2)$ \\
\hline & & Aplicabilidade & 48 & $38(79,2)$ & $10(20,8)$ & & $48(100)$ & \\
\hline & & Objetividade & 48 & $35(72,9)$ & $12(25)$ & $1(2,1)$ & & $1(2,1)$ \\
\hline & & Conteúdo & 48 & $35(72,9)$ & $12(25)$ & $1(2,1)$ & & $1(2,1)$ \\
\hline \multirow{4}{*}{$\begin{array}{l}\text { Ocupacional - } \\
\text { Riscos físicos, } \\
\text { quimicos, biológios } \\
\text { e ergonômicos ( } 14 \\
\text { e 15) }\end{array}$} & \multirow{4}{*}{6} & Clareza & 12 & $6(50)$ & $5(41,7)$ & $1(8,3)$ & $12(100)$ & \\
\hline & & Aplicabilidade & 12 & $9(75)$ & $2(16,7)$ & $1(8,3)$ & $12(100)$ & \\
\hline & & Objetividade & 12 & $7(58,3)$ & $4(33,3)$ & $1(8,3)$ & $12(100)$ & \\
\hline & & Conteúdo & 12 & $9(75)$ & $2(16,7)$ & $1(8,3)$ & $12(100)$ & \\
\hline $\begin{array}{l}\text { Ocupacional- } \\
\text { Estresse e ambiente } \\
\text { de trabalho } \\
(16 \text { a } 18) \\
\end{array}$ & 6 & $\begin{array}{l}\text { Clareza, } \\
\text { Aplicabilidade, } \\
\text { Objetividade e } \\
\text { Conteúdo } \\
\end{array}$ & 18 & $11(61,1)$ & $6(33,3)$ & $1(5,6)$ & $17(94,4)$ & $1(5,6)$ \\
\hline \multirow{4}{*}{$\begin{array}{l}\text { Ocupacional - } \\
\text { Doenças e Acidente } \\
\text { de Trabalho (19 a 22) }\end{array}$} & \multirow{4}{*}{6} & Clareza & 24 & $19(72,9)$ & $5(20,8)$ & & $24(100)$ & \\
\hline & & Aplicabilidade & 24 & $24(100)$ & - & & $24(100)$ & \\
\hline & & Objetividade & 24 & $22(91,7)$ & $2(8,3)$ & & $24(100)$ & \\
\hline & & Conteúdo & 24 & $22(91,7)$ & $2(8,3)$ & & $24(100)$ & \\
\hline \multirow{4}{*}{$\begin{array}{l}\text { Cardiovascular } \\
(23 \text { a } 27)\end{array}$} & \multirow{4}{*}{6} & Clareza & 30 & $22(73,3)$ & $7(23,3)$ & $1(3,3)$ & & $1(3,3)$ \\
\hline & & Aplicabilidade & 30 & $22(73,3)$ & $8(26,7)$ & & $30(100)$ & \\
\hline & & Objetividade & 30 & $20(66,7)$ & $9(30)$ & $1(3,3)$ & $30(100)$ & \\
\hline & & Conteúdo & 30 & $22(73,3)$ & $8(26,7)$ & & $30(100)$ & \\
\hline $\begin{array}{l}\text { Osteomuscular } \\
(28 \text { a } 30)\end{array}$ & 6 & $\begin{array}{l}\text { Clareza, } \\
\text { Aplicabilidade, } \\
\text { Objetividade e } \\
\text { Conteúdo }\end{array}$ & 18 & $13(72,2)$ & $5(27,8)$ & & $18(100)$ & \\
\hline
\end{tabular}

*Valores referentes ao $\mathrm{n}^{\circ}$ de avaliadores $\mathrm{X} \mathrm{n}^{\circ}$ de questões de cada núcleo 
Chaise FO, et al. Validade e reprodutibilidade do Questionário de Hábitos. Rev Ter Ocup Univ São Paulo. 2016 maio/ago.;27(2):199-215.

Tabela 2 - Modificações do QHViT propostas pelos especialistas/avaliadores

\begin{tabular}{|c|c|c|}
\hline Modificações & $1^{\text {a }}$ Versão & $2^{\mathrm{a}}$ Versão $=$ Versão Final \\
\hline \multirow{3}{*}{$\begin{array}{l}\text { Nome do } \\
\text { núcleo }\end{array}$} & DEMOGRÁFICO & IDENTIFICAÇÃO \\
\hline & $\begin{array}{l}\text { OCUPACIONAL } \\
\text { Jornada de Trabalho }\end{array}$ & $\begin{array}{l}\text { OCUPACIONAL } \\
\text { História Laboral e Jornada de Trabalho }\end{array}$ \\
\hline & $\begin{array}{l}\text { OCUPACIONAL } \\
\text { Estresse e Ambiente de Trabalho }\end{array}$ & $\begin{array}{l}\text { OCUPACIONAL } \\
\text { Estresse, Organização e Relações de Trabalho }\end{array}$ \\
\hline \multirow{9}{*}{ Questões } & Qual a sua cor? & $\begin{array}{l}\text { Qual sua cor? } \\
\text { ( ) branca ( ) preta ( ) parda } \\
\text { ( ) Amarela/indígena }\end{array}$ \\
\hline & $\begin{array}{l}\text { Qual a sua escolaridade? } \\
\text { ( ) primário completo ( ) primário incompleto } \\
\text { ( ) } 1^{\circ} \text { grau completo ( ) } 1^{\circ} \text { grau incompleto } \\
\text { ( ) } 2^{\circ} \text { grau completo ( ) } 2^{\circ} \text { grau incompleto } \\
\text { ( ) superior completo ( ) superior incompleto }\end{array}$ & $\begin{array}{l}\text { Qual a sua escolaridade? } \\
\text { ( ) Ensino Fundamental Completo } \\
\text { ( ) Ensino Fundamental Incompleto } \\
\text { ( ) Ensino Médio Completo ( } 2^{\circ} \text { grau) } \\
\text { ( ) Ensino Médio Incompleto ( } 2^{\circ} \text { grau) } \\
\text { ( ) Ensino Superior Completo } \\
\text { ( ) Ensino Superior Incompleto } \\
\text { ( ) Pós-Graduação Completa } \\
\text { ( ) Pós-Graduação em andamento }\end{array}$ \\
\hline & Qual a sua função desempenhada neste serviço? & Qual o seu cargo atualmente? \\
\hline & Há quanto tempo você trabalha nesta função? & Há quanto tempo você trabalha nesse cargo? \\
\hline & Há quanto tempo você trabalha na área da saúde? & Há quanto tempo você trabalha neste serviço? \\
\hline & Qual é sua carga horária mensal? & $\begin{array}{l}\text { Qual é sua carga horária mensal (incluindo todos seus vínculos } \\
\text { de trabalho)? }\end{array}$ \\
\hline & $\begin{array}{l}\text { Você costuma fazer horas extras? } \\
\text { ( ) Não ( ) Sim ( ) Quantas? }\end{array}$ & $\begin{array}{l}\text { No último mês você fez horas extras neste serviço? } \\
\text { ( ) Não ( ) Sim ( ) Quantas? }\end{array}$ \\
\hline & $\begin{array}{l}\text { No último ano você teve diagnóstico de: } \\
\text { ( ) Depressão ( ) Síndrome do Pânico } \\
\text { ( ) Transtorno de Ansiedade ( ) Transtorno Bipolar } \\
\text { ( ) Nenhum dos problemas relacionados } \\
\text { ( ) Outro, qual? }\end{array}$ & $\begin{array}{l}\text { No último ano você teve algum(ns) problema(s) de saúde } \\
\text { mental? Qual (is)? }\end{array}$ \\
\hline & $\begin{array}{l}\text { No último ano você teve algum diagnóstico de } \\
\text { doença de osteomuscular? } \\
\text { ( ) Hérnia de Disco ( ) Artrose ( ) Artrite } \\
\text { ( ) Bursite ( ) Tendinite ( ) Sinovite } \\
\text { ( ) Tenossinovite ( ) Epicondilite } \\
\text { ( ) Outra, qual? }\end{array}$ & $\begin{array}{l}\text { No último ano você teve algum distúrbio osteomusculares } \\
\text { relacionado ao trabalho? } \\
\text { ( ) não ( ) sim } \\
\text { Se sim, marque qual dos distúrbios osteomusculares listados } \\
\text { abaixo você teve no último ano? } \\
\text { ( ) Hérnia de Disco ( ) Artrose } \\
\text { ( ) Bursite ( ) Tendinite } \\
\text { ( ) Sinovite ( ) Tenossinovite } \\
\text { () Epicondilite () Outro, qual? }\end{array}$ \\
\hline \multirow{3}{*}{$\begin{array}{l}\text { Inserção de } \\
\text { questão }\end{array}$} & & $\begin{array}{l}\text { Houve variação no seu peso desde o início da função até os dias } \\
\text { de hoje? } \\
\text { ( ) não alterou ( ) aumentou, quantos } \mathrm{Kg} \text { ? ( ) diminuiu, quantos } \\
\mathrm{Kg} \text { ? }\end{array}$ \\
\hline & & $\begin{array}{l}\text { Você possui outro vínculo de trabalho? } \\
\begin{array}{ll}\text { ( ) não } & \text { ( ) sim }\end{array}\end{array}$ \\
\hline & & $\begin{array}{l}\text { Você tem pausa/descanso durante a execução de suas tarefas no } \\
\text { seu serviço? } \\
\text { ( ) Nunca ( ) Algumas vezes ()Sempre }\end{array}$ \\
\hline
\end{tabular}


Tabela 3 - Características socio-demográficas dos participantes da etapa de reprodutibilidade

\begin{tabular}{|c|c|c|c|c|c|}
\hline & \multicolumn{5}{|l|}{ Função } \\
\hline & $\begin{array}{l}\text { Condutores de } \\
\text { Ambulância } \\
(n=14)\end{array}$ & $\begin{array}{c}\text { Técnicos de } \\
\text { Enfermagem }(n=13)\end{array}$ & $\begin{array}{l}\text { Enfermeiros } \\
\qquad(n=4)\end{array}$ & $\begin{array}{l}\text { Médicos } \\
(n=6)\end{array}$ & $\begin{array}{c}\text { Telefonistas } \\
(\mathrm{n}=6)\end{array}$ \\
\hline \multicolumn{6}{|l|}{ Sexo } \\
\hline Masculino \% (n) & $100(14)$ & $53,8(7)$ & & $50(3)$ & $16,7(1)$ \\
\hline Feminino \% (n) & & $46,2(6)$ & $100(4)$ & $50(3)$ & $83,3(5)$ \\
\hline Idade $(\operatorname{anos}) \bar{x}( \pm d p)$ & $48,5(8,0)$ & $39,2(7,98)$ & $32,2(6,18)$ & $37,1(4,79)$ & $41,1(14,2)$ \\
\hline $\operatorname{Altura}(\mathrm{m}) \bar{x}( \pm \mathrm{dp})$ & $1,71(7,78)$ & $1,55(41,8)$ & $1,63(8,9)$ & $1,71(8,3)$ & $1,64(4,6)$ \\
\hline $\operatorname{Peso}(\mathrm{kg}) \bar{x}( \pm \mathrm{dp})$ & $83(16,4)$ & $80(17,7)$ & $71,7(13)$ & $74,4(9,7)$ & $78(15,2)$ \\
\hline \multicolumn{6}{|l|}{ Escolaridade \% (n) } \\
\hline Ensino Fundamental Completo & $28,6(4)$ & & & & \\
\hline Ensino Fundametal Incompleto & $14,3(2)$ & & & & \\
\hline Ensino Médio Completo & $28,6(4)$ & $61,5(8)$ & & & $50(3)$ \\
\hline Ensino Médio Incompleto & $14,3(2)$ & & & & \\
\hline Ensino Superior Completo & $7,1(1)$ & $7,7(1)$ & $25(1)$ & & $33,3(2)$ \\
\hline Ensino Superior Incompleto & $7,1(1)$ & $30,8(4)$ & & & $16,7(1)$ \\
\hline Pós-Graduação Completa & & & $75(3)$ & $83,3(5)$ & \\
\hline Pós-Graduação em andamento & & & & $16,7(1)$ & \\
\hline
\end{tabular}

Tabela 4 - Valores de reprodutibilidade do QHT (n=43)

\begin{tabular}{|c|c|c|c|}
\hline Núcleo & Questões & $\% \mathrm{C}$ & $\mathbf{k}$ \\
\hline Identificação & $\begin{array}{l}\text { 5. Houve variação no seu peso desde o início da função até os dias } \\
\text { de hoje? }\end{array}$ & 95,3 & $0,89(0,709-1,000)$ \\
\hline \multirow{6}{*}{$\begin{array}{l}\text { Ocupacional HLJT } \\
\text { - História Laboral e } \\
\text { Jornada de Trabalho }\end{array}$} & 11. Qual é o seu regime de trabalho neste serviço? & 95,3 & $0,88(0,739-1,000)$ \\
\hline & 12. No último mês você fez horas extras neste serviço? & 97,6 & $0,919(0,697-1,000)$ \\
\hline & 12. Quantas horas extras você fez neste serviço no ultimo mês? & 82,1 & $0,792(0,618-0,955)$ \\
\hline & 13. Quais são seus turnos de trabalho habituais neste serviço? & 83,7 & $0,790(0,673-0,913)$ \\
\hline & 14. Você possui outro vínculo de trabalho? & 97,6 & $0,941(0,790-1,000)$ \\
\hline & $\begin{array}{l}\text { 15. Qual é sua carga horária mensal (incluindo todos seus vínculos } \\
\text { de trabalho)? }\end{array}$ & 82,1 & $0,792(0,615-0,919)$ \\
\hline \multirow{16}{*}{$\begin{array}{l}\text { Ocupacional RFQBE } \\
\text { - Riscos Físicos, } \\
\text { Químicos, Biológicos e } \\
\text { Ergonômicos }\end{array}$} & $\begin{array}{l}\text { 16. Marque com um X com que frequência você tem contato/ex- } \\
\text { posição no desempenho de sua função }\end{array}$ & & \\
\hline & Frio/Calor excessivo & 88,3 & $0,748(0,519-0,945)$ \\
\hline & Ruído & 86,0 & $0,501(0,096-0,801)$ \\
\hline & Vibrações & 90,4 & $0,808(0,589-0,955)$ \\
\hline & Sangue & 83,7 & $0,705(0,472-0,890)$ \\
\hline & Secreções & 83,7 & $0,719(0,525-0,886)$ \\
\hline & Perfuro-cortante & 86,0 & $0,748(0,520-0,916)$ \\
\hline & 17. Marque um X com que frequência sua função exige: & & \\
\hline & movimentos repetitivos & 83,7 & $0,620(0,351-0,849)$ \\
\hline & Rapidez na tarefa & 95,3 & $0,645(0,365-0,876)$ \\
\hline & Esforço Físico & 88,3 & $0,801(0,626-0,958)$ \\
\hline & Trabalho sentado & 88,3 & $0,775(0,531-0,922)$ \\
\hline & Trabalho em pé & 81,3 & $0,674(0,445-0,849)$ \\
\hline & Concentração na tarefa & 93,0 & $0,696(0,261-1,000)$ \\
\hline & Uso do computador & 88,3 & $0,823(0,648-0,930)$ \\
\hline & Atuação em diferentes espaços & 88,3 & $0,765(0,548-0,945)$ \\
\hline
\end{tabular}


Tabela 4 - Valores de reprodutibilidade do QHT $(n=43)$

\begin{tabular}{|c|c|c|c|}
\hline Núcleo & Questões & $\% \mathrm{C}$ & $\mathbf{k}$ \\
\hline \multirow{10}{*}{$\begin{array}{l}\text { Ocupacional EORT - } \\
\text { Estresse, Organização } \\
\text { e Relações de Trabalho }\end{array}$} & $\begin{array}{l}\text { 18. Marque com um } \mathrm{X} \text { a frequência que você se sente estressado no } \\
\text { trabalho em relação a: }\end{array}$ & & \\
\hline & Tomada de decisão & 95,3 & $0,856(0,642-1,000)$ \\
\hline & Tempo e velocidade na realização do trabalho & 83,7 & $0,701(0,476-0,906)$ \\
\hline & Trabalho em equipe & 81,4 & $0,729(0,536-0,890)$ \\
\hline & Relação com a chefia & 95,3 & $0,921(0,797-1,000)$ \\
\hline & Remuneração & 83,7 & $0,733(0,530-0,889)$ \\
\hline & Jornada de Trabalho & 76,7 & $0,627(0,400-0,815)$ \\
\hline & Pressão para a produtividade & 81,4 & $0,675(0,463-0,853)$ \\
\hline & Restrição de sono & 88,3 & $0,810(0,635-0,961)$ \\
\hline & $\begin{array}{l}\text { 19. Você tem pausa/descanso durante a execução de suas tarefas no } \\
\text { seu serviço? }\end{array}$ & 93,0 & $0,860(0,664-1,000)$ \\
\hline $\begin{array}{l}\text { Ocupacional DAT - } \\
\text { Doenças e Acidentes } \\
\text { de Trabalho }\end{array}$ & $\begin{array}{l}\text { 24. Durante o desempenho de sua função você já sofreu algun aci- } \\
\text { dente de trabalho? }\end{array}$ & 86,0 & $0,853(0,723-0,943)$ \\
\hline \multirow[t]{2}{*}{ Cardiovascular } & $\begin{array}{l}\text { 28. Você possui parente de primeiro grau masculino com menos de } \\
55 \text { anos ou feminino com menos de } 65 \text { anos que teve doença } \\
\text { cardíaca ou acidente vascular cerebral? }\end{array}$ & 93,0 & $0,860(0,676-1,000)$ \\
\hline & 30. Você realiza algum exercício físico? & 97,6 & $0,948(0,823-1,000)$ \\
\hline \multirow{4}{*}{ Osteomuscular } & $\begin{array}{l}\text { 31. No último ano você teve algum distúrbio osteomusculares rela- } \\
\text { cionado ao trabalho? }\end{array}$ & 93 & $0,861(0,682-1,000)$ \\
\hline & $\begin{array}{l}\text { 32. Nos últimos três meses, você apresentou dor em alguma região } \\
\text { do corpo. Qual a intensidade? }\end{array}$ & 81,4 & $0,762(0,519-0,948)$ \\
\hline & $\begin{array}{l}\text { 32. Nos últimos três meses, você apresentou dor em alguma região } \\
\text { do corpo. Qual a frequência dessa dor? }\end{array}$ & 86,0 & $0,780(0,491-1,000)$ \\
\hline & 33. Você faz uso de medicamentos para essa dor ou desconforto? & 93,0 & $0,860(0,677-1,000)$ \\
\hline
\end{tabular}

Apenas com intuito de identificar o perfil da amostra avaliada, os resultados de prevalência e fatores de risco da amostra estudada, utilizando o QHT, mostraram que: (1) a prevalência de HAS foi de $55,8 \%$; (2) a prevalência de dor musculoesquelética foi de $92,9 \%$; (3) a HAS e a obesidade foram referidos como fatores de risco para as DCV; e (4) o esforço físico, a não realização de pausa no trabalho e a jornada de trabalho de 12 horas como fatores de risco para as DORT's.

\section{DISCUSSÃO}

Para a realização dos propósitos do estudo foi necessário que o QHT passasse pelas etapas descritas na literatura: revisão da literatura sobre o assunto, observação de campo, validade de conteúdo e a reprodutibilidade do instrumento. Os instrumentos devem ser desenvolvidos a partir da revisão da literatura sobre o tema em questão ou até mesmo a partir de resultados de pesquisas qualitativas, nas quais representantes da população estudada fornecem dados sobre a experiência vivenciada ${ }^{19}$. Na Etapa 1 do presente trabalho procuramos atender tanto a revisão teórica sobre o tema, quanto por meio da observação de campo, despertando-nos para os fenômenos de interesse ${ }^{20}$.

A Etapa 3 de validade de conteúdo consistiu em verificar se os itens propostos pelo QHT constituemse numa amostra representativa do que se deseja medir, ou seja, em que proporção os itens do instrumento servem para medir a construção do todo ${ }^{20}$. Sendo assim, especialistas no assunto avaliaram quantitativamente ou qualitativamente o $\mathrm{QHT}^{21,22}$. Para esta avaliação, a literatura é controversa, podendo variar de cinco a 10 avaliadores $^{21}$ ou ainda entre seis e 20 especialistas $^{23}$. A solidez do processo de validação pode ser influenciada pela experiência e know-how dos especialistas no tema do instrumento ${ }^{19}$. São sugeridos critérios para que sejam convidados os experts, como as publicações em revistas indexadas e a experiência clínica ${ }^{19}$. Considerando que no presente estudo participaram seis especialistas que possuíam publicações em revistas e experiência no tema, entendemos que isso contribuiu para a solidez do processo de validação de conteúdo do QHT.

A literatura comenta sobre a subjetividade para avaliar a validade de conteúdo. A falta de medidas objetivas 
indica que não há consenso sobre a extensão que determina que a medida/item/questão tenha atingido a validade de seu conteúdo ${ }^{20}$. Para solucionar esse problema, tem sido sugerido que a avaliação da medida/item/questão deva ser realizada utilizando-se uma escala do tipo Likert, que fornece medidas quantitativas de conteúdo à medida que os especialistas concordam sobre a relevância de conteúdo ${ }^{24}$. Os critérios estabelecidos para que os juízes avaliem o instrumento são importantes na fase de validação de conteúdo. Entre esses critérios estão a representatividade, a qual consiste na capacidade de um item para representar o domínio de conteúdo; clareza, a qual demonstra o quão claro o item está formulado; a estrutura do instrumento e a abrangência de conteúdo dos itens ${ }^{20}$.

Para a avaliação de conteúdo do QHT esses critérios foram avaliados, utilizando uma escala Likert (excelente, bom e insuficiente) e analisados quantitativamente através de estatística descritiva por meio de tabelas de frequência. Após a primeira rodada de avaliação, o QHT necessitou de alterações propostas pelos avaliadores e evidenciadas por meio das frequências. Já na segunda rodada de avaliação, os itens foram avaliados em sua maioria como excelentes, considerando o QHT válido em seu conteúdo.

$\mathrm{Na}$ Etapa 4 foi testada a concordância entre as respostas dadas pelos trabalhadores da saúde em dois dias distintos, por meio do $\% \mathrm{C}$ e do valor de $\mathrm{k}$, apresentando concordâncias consideradas boas e muito boas. Cabe comentar que mesmo na questão $18 \mathrm{em}$ que o trabalhador deve indicar a frequência de seu estresse frente à organização, ao ambiente e às relações de trabalho, a concordância foi considerada boa. Esse tipo de questão depende da percepção do respondente nos dias da aplicação do instrumento, que pode estar alterada pela alta demanda de trabalho naquele período ou por qualquer motivo pessoal, o que poderia ter afetado negativamente a reprodutibilidade ${ }^{25}$.

A relevância do presente estudo reside na apresentação de um instrumento validado em seu conteúdo, que contempla a avaliação dos fatores de risco (cardiovasculares e osteomusculares) para o adoecimento de trabalhadores da área da saúde, o qual poderá ser utilizado amplamente pelos serviços para a realização de avaliações periódicas, bem como para o mapeamento do comportamento dos fatores de risco ao longo do tempo. Essas informações permitem a adoção de práticas transformadoras das condições e situações de trabalho pelas instituições. Ainda, como vantagem do QHT, citamos a facilidade da obtenção das informações, estando reunidas em um único instrumento. Assim, tornase atrativo seu uso no ambiente da pesquisa cientifica (que visa, por exemplo, apontar quais aspectos do trabalho podem comprometer a saúde dos trabalhadores) e na prática institucional (que visa, por exemplo, melhorar as condições no trabalho). A utilização de um único instrumento também facilita a reprodução e a comparação de estudos desenvolvidos em diferentes contextos. Até o momento, estudos que visassem avaliar os hábitos de trabalho dos trabalhadores da saúde necessitavam utilizar uma associação de vários instrumentos.

Por exemplo, em um estudo anterior ${ }^{25}$, cujo intuito foi avaliar a saúde mental e bem-estar emocional dos trabalhadores escoceses em atendimento pré-hospitalar móvel, utilizou-se o General Health Questionaire-28, que identifica transtornos psiquiátricos, associado ao Impact of Event Scale, que determina a frequência de sintomas de pós-traumáticos em relação a eventos críticos específicos. Em outro estudo ${ }^{12}$ a saúde mental e física dos profissionais do SAMU 192 de João Pessoa/PB foi avaliada com um questionário sociodemográfico associado ao Índice de Capacidade para o Trabalho, adaptado e validado para o Brasil. Em mais um exemplo, Patterson et al. ${ }^{11}$ avaliaram a qualidade do sono associado à fadiga entre médicos e paramédicos do serviço de emergencia na Pensilvânia, com dois questionários Pittsburgh Sleep Quality Index e Chalder Fatigue Questionnaire, respectivamente. Esses exemplos nos inspiraram a desenvolver o QHT, um instrumento que permite identificar os hábitos relacionados ao trabalho, englobando aspectos demográficos e ocupacionais e a saúde osteomuscular e cardiovascular, especialmente desenvolvido para profissionais da saúde.

Como "limitações do instrumento" citamos: (1) a não inclusão do fator de risco etilismo apontado na literatura; (2) a não inclusão de questões especificas sobre a demanda de trabalho em relação aos recursos humanos, que possibilitasse contemplar as especificidades de cada profissional e identificar a existência ou não de desequilíbrio na relação profissional e demanda de trabalho; (3) a não inclusão de questões especificas sobre a qualidade e disponibilidade dos recursos estruturais, técnicos e humanos para o desenvolvimento das atividades do trabalho; e (4) a falta de questões abertas no núcleo ocupacional que permitiriam a compreensão das reais condições e situações de trabalho em toda a sua complexidade. Enquanto "limitações do estudo" podemos citar: (1) as demandas do serviço no momento da aplicação do QHT, sendo que durante a coleta de dados alguns trabalhadores necessitaram parar o preenchimento para atender aos chamados de urgência próprios do serviço; (2) a não utilização de grau de concordância entre especialistas quanto aos itens a serem mantidos ou retirados do instrumento; (3) a falta de um especialista da 
área da saúde do trabalhador no processo de validação de conteúdo; e (4) a falta de informação sobre a aplicabilidade e a abrangência do instrumento em si.

Assim, identificamos a necessidade de futuros estudos com intuito de suprir essas limitações do QHT e ampliarem a validade do instrumento, bem como registrar sua aplicação em pesquisas observacionais ou de intervenção na área da saúde. Entendemos que o alcance prático do QHT está na sua facilidade em obtenção de informações para conhecer o perfil e alguns importantes aspectos de rotina de trabalho dos trabalhadores da saúde, útil para avaliação da saúde do trabalhador, bem como para profissionais da Terapia Ocupacional. Não obstante, aconselhamos que a utilização do QHT seja complementada com entrevistas e/ou grupos focais, de modo a garantir a qualidade e a efetividade de pesquisa e intervenção em fase inicial.

\section{CONCLUSÃO}

Considerando que foram seguidas todas as etapas necessárias para o desenvolvimento de um instrumento de avaliação, concluímos que o QHT é apropriado para trabalhadores da área da saúde, pois apresenta validade de conteúdo e reprodutibilidade. Sendo assim, o QHT pode ser utilizado em estudos que visem avaliar hábitos relacionados ao trabalho e fatores de risco associados para o aparecimento de DCV e DORT's. Ainda, cabe ressaltar que a proposta de uso do QHT não se restringe somente à pesquisa, mas também pode ser utilizado na prática clínica para promover a saúde do trabalhador.

Colaboração dos autores: FO Chaise trabalhou na concepção dos dados e na redação do artigo; AP Kasten e J Pasa - trabalharam na concepção e análise dos dados; TS Furlanetto e CT Candotti - trabalharam na redação do artigo, revisão crítica e na aprovação da versão a ser publicada. 


\section{APÊNDICE 1 - QUESTIONÁRIO DE HÁBITOS DE VIDA NO TRABALHO (QHT)}

\section{NÚCLEO IDENTIFICAÇÃO - Q1 ATÉ Q6}

Sexo:
( ) Masculino
( ) Feminino

1. Qual sua idade?

2. Qual sua cor?
( ) branca
() preta
( ) parda
( ) amarela/indígena

3. Qual a sua altura $(\mathrm{m})$ ?

4. Qual seu peso $(\mathrm{Kg})$ ?

5. Houve variação no seu peso desde o início da função até os dias de hoje?

( ) não alterou ( ) aumentou, quantos $\mathrm{Kg}$ ?

( ) diminuiu, quantos $\mathrm{Kg}$ ?

6. Qual a sua escolaridade?

( ) Ensino Fundamental Completo ( $\left(1^{\circ} \mathrm{grau}\right)$

( ) Ensino Fundamental Incompleto ( $1^{\circ}$ grau $)$

( ) Ensino Médio Completo ( $2^{\circ}$ grau)

( ) Ensino Médio Incompleto ( $\left.2^{\circ} \mathrm{grau}\right)$

( ) Ensino Superior Completo

( ) Ensino Superior Incompleto

( ) Pós-Graduação Completa

( ) Pós-Graduação em andamento

\section{NÚCLEO OCUPACIONAL HLJT - História Laboral e Jornada de Trabalho - Q7 À Q15}

7. Qual sua profissão?

8. Qual o seu cargo atualmente?

9. Há quanto tempo você trabalha nesse cargo?

10. Há quanto tempo você trabalha neste serviço?

11. Qual é o seu regime de trabalho neste serviço?

( ) 6 horas diárias

( ) 8 horas diárias

( ) plantão de 12 horas

( ) plantão de 24 horas

( ) outro, qual?

12. No último mês você fez horas extras neste serviço?
( ) Não
( ) $\mathrm{Sim}$
( ) Quantas? 
13. Quais são seus turnos de trabalho habituais neste serviço?
( ) Manhã
( ) Tarde
( ) Noite

( )Alternado/intercalado, como?

14. Você possui outro vínculo de trabalho?
( ) não
( ) $\operatorname{sim}$

15. Qual é sua carga horária mensal (incluindo todos seus vínculos de trabalho)?

\section{NÚCLEO OCUPACIONAL RFQBE - Riscos Físicos, Químicos, Biológicos e Ergonômicos - Q16 e Q17}

16. Marque com um $\mathbf{X}$ com que frequência você tem contato/exposição com os itens abaixo listados no desempenho de sua função:

\begin{tabular}{|l|l|l|l|}
\hline Contato/Exposição & Nunca & $\begin{array}{l}\text { Algumas } \\
\text { Vezes }\end{array}$ & Sempre \\
\hline Calor excessivo & & & \\
\hline Frio excessivo & & & \\
\hline Ruído/Barulho & & & \\
\hline Vibrações & & & \\
\hline Sangue & & & \\
\hline Secreções & & & \\
\hline Material perfuro-cortante & & & \\
\hline Outro? & & & \\
\hline
\end{tabular}

17. Marque um $\mathbf{X}$ com que frequência sua função exige:

\begin{tabular}{|l|l|l|l|}
\hline Exigência da Função & Nunca & $\begin{array}{l}\text { Algumas } \\
\text { Vezes }\end{array}$ & Sempre \\
\hline Movimentos repetitivos & & & \\
\hline Rapidez na execução de tarefas & & & \\
\hline Esforço físico & & & \\
\hline Trabalho sentado & & & \\
\hline Trabalho em pé & & & \\
\hline $\begin{array}{l}\text { Concentração/Atenção exclusiva } \\
\text { na tarefa }\end{array}$ & & & \\
\hline Uso do computador & & & \\
\hline $\begin{array}{l}\text { Atuação em diferentes espaços } \\
\text { físicos de trabalho durante a tarefa } \\
\text { desempenhada no seu serviço }\end{array}$ & & & \\
\hline Outra? & & & \\
\hline
\end{tabular}




\section{NÚCLEO OCUPACIONAL EORT - Estresse, Organização e Relações de Trabalho - Q18 À 21}

18. Marque com um $\mathbf{X}$ a frequência que você se sente estressado no trabalho em relação a:

\begin{tabular}{|l|l|l|l|}
\hline $\begin{array}{l}\text { Organização e Relações } \\
\text { de Trabalho }\end{array}$ & Nunca & $\begin{array}{c}\text { Algumas } \\
\text { Vezes }\end{array}$ & Sempre \\
\hline $\begin{array}{l}\text { Tomada de } \\
\text { decisão }\end{array}$ & & & \\
\hline $\begin{array}{l}\text { Tempo e velocidade na } \\
\text { realização do trabalho }\end{array}$ & & & \\
\hline Trabalho em equipe & & & \\
\hline Relação com a chefia & & & \\
\hline Remuneração & & & \\
\hline $\begin{array}{l}\text { Jornada de } \\
\text { trabalho }\end{array}$ & & & \\
\hline $\begin{array}{l}\text { Pressão para produtivi- } \\
\text { dade }\end{array}$ & & & \\
\hline Restrição do Sono & & & \\
\hline
\end{tabular}

19. Você tem pausa/descanso durante a execução de suas tarefas no seu serviço?
( ) Nunca
( ) Algumas vezes
( ) Sempre

20. No último ano você teve algum(ns) problema(s) de saúde mental? Qual (is)?

21. Você faz uso de medicamentos para esse problema?
( ) não
( ) sim, qual (is) medicamentos?

\section{NÚCLEO OCUPACIONAL DAT - Doenças e Acidentes de Trabalho - Q22 À Q25}

22. Você já se afastou do trabalho por motivo de doença?
( ) não
( ) sim, qual(is) a(s) doença(s)?

23. Quanto tempo (em dias) no último ano, você se afastou do trabalho por motivo de doença?

24. Durante o desempenho de sua função você já sofreu:

( ) Acidentes de trabalho com material pérfuro-cortante

( ) Agressão moral

( ) Contaminação por substâncias químicas

( ) Agressão física

( ) Contaminação por substâncias biológicas

( ) Acidente automobilístico

( ) Nenhum dos eventos relacionados

( ) Outro, qual?

25. No último ano, quantas vezes você sofreu algum desses acidentes do trabalho? 


\section{NÚCLEO CARDIOVASCULAR - Q26 À Q30}

26. Marque com um $\mathbf{X}$ os sintomas e as doenças cardiovasculares relacionadas abaixo:

\begin{tabular}{|l|l|l|l|}
\hline $\begin{array}{l}\text { Sintomas e Doenças } \\
\text { Cardiovasculares }\end{array}$ & $\begin{array}{c}\text { Você já } \\
\text { teve }\end{array}$ & $\begin{array}{l}\text { Você teve e } \\
\text { Ainda tem }\end{array}$ & $\begin{array}{c}\text { Você } \\
\text { tem }\end{array}$ \\
\hline Angina Estável & & & \\
\hline Angina Instável & & & \\
\hline Insuficiência cardíaca & & & \\
\hline Aneurisma & & & \\
\hline Infarto & & & \\
\hline Arritmia cardíaca & & & \\
\hline AVC & & & \\
\hline Colesterol Alto & & & \\
\hline Diabetes & & & \\
\hline $\begin{array}{l}\text { Hipertensão } \\
\text { Arterial Sistêmica }\end{array}$ & & & \\
\hline Outra? & & & \\
\hline
\end{tabular}

27. Você faz tratamento medicamentoso para alguma dessas doenças mencionadas acima?

( ) não ( ) sim, que medicamentos utiliza?

28. Você possui parente de primeiro grau masculino com menos de 55 anos ou feminino com menos de 65 anos que teve doença cardíaca ou acidente vascular cerebral?
( ) não
( ) $\operatorname{sim}$

29. Você é fumante?

( ) não ( ) sim, há quanto tempo fuma?

quantos cigarros por dia?

( ) parou de fumar, há quanto tempo parou?

30. Você realiza algum exercício físico?

( ) não $\quad$ ( ) sim, qual?há quanto tempo?

Se sim, com que frequência você realiza esse exercício físico?
( ) $1 \mathrm{vez} /$ semana
( ) 2 vezes/semana
( ) 3 vezes/semana
( ) 4 vezes/semana
( ) 5 vezes/semana
( ) todos os dias da semana 


\section{NÚCLEO OSTEOMUSCULAR - Q31 À Q33}

31. No último ano você teve algum distúrbio osteomusculares relacionado ao trabalho?

( ) não

( ) $\operatorname{sim}$

Se sim, marque qual dos distúrbios osteomusculares listados abaixo você teve no último ano?
( ) Hérnia de Disco
( ) Artrose
( ) Bursite
() Tendinite
() Sinovite
() Tenossinovite
() Epicondilite
() Outro, qual?

32. Nos últimos três meses, você apresentou dor em alguma região do corpo? Qual a intensidade e frequência dessa dor? Marque com um X na tabela abaixo:

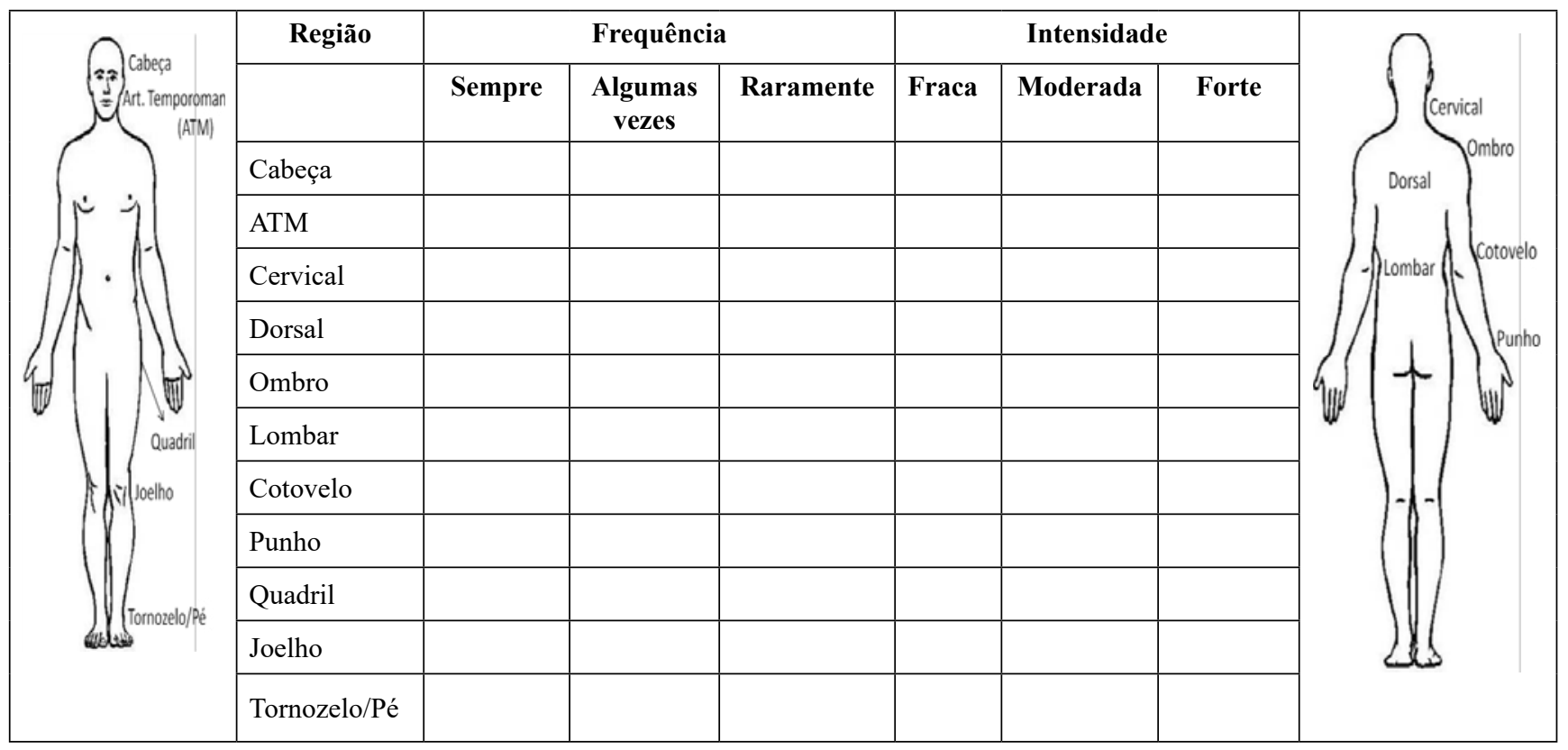

33. Você faz uso de medicamentos para essa dor ou desconforto?
( ) não
( ) sim, qual (is) medicamento (s)?

Qual a frequência de uso e a dosagem (mg, via oral, intramuscular, etc)?

Obrigada pela participação! 


\section{REFERÊNCIAS}

1. Couto HDA, Vieira FLH, Lima EG. Estresse ocupacional e hipertensão arterial sistêmica. Rev Bras Hipertens. 2007;14(2):112-5.

2. Magnago TSBDS, Lisboa MTL, Griep RH. Trabalho da enfermagem e distúrbio musculoesquelético: revisão das pesquisas sobre o tema. Esc Anna Nery. 2008;12:560-5. DOI: $10.1590 / \mathrm{S} 1414-81452008000300025$.

3. Célia RDCRDS, Alexandre NMC. Distúrbios osteomusculares e qualidade de vida em trabalhadores envolvidos com transporte de pacientes. Rev Bras Enferm. 2003;56:494-8. DOI: 10.1590/S003471672003000500005 .

4. Monteiro MS, Alexandre NMC, Rodrigues CM. Doenças músculo-esqueléticas, trabalho-esqueléticas, trabalho e estilo de vida entre trabalhadores de estilo de uma instituição pública de saúde. Rev Esc Enferm USP. 2006;40:20-5. DOI: 10.1590/S0080-62342006000100003.

5. JardimTDSV, Jardim PCSBOV, Araújo WECD, Jardim LMSSV, Salgado CM. Fatores de risco cardiovasculares em coorte de profissionais da Área médica: 15 anos de evolução. Arq Bras Cardiol. 2010;95:332-8. DOI: 10.1590/ S0066-782X2010005000084.

6. Maia CO, Goldmeier S, Moraes MA, Boaz MR, Azzolin $\mathrm{K}$. Fatores de risco modificáveis para doença arterial coronariana nos trabalhadores de enfermagem. Acta Paul Enferm. 2007;20(2):138-42. DOI: 10.1590/S010321002007000200005.

7. Reis ALC, Fernandes SN, Gomes AF. Estresse e fatores psicossociais. Psicol Cien Profissão. 2010;30:712-25. DOI: $10.1590 /$ S1414-98932010000400004.

8. Berger W, Figueira I, Maurat AM, Bucassio EP, Vieira I, Jardim SR, Coutinho ES, Mari JJ, Mendlowicz MV. Partial and full ptsd in brazilian ambulance workers: prevalence and impact on health and on quality of life. J Trauma Stress. 2007;20(4):637-42. DOI: 10.1002/jts.20242.

9. Vegian CFLO, Monteiro MIS. Living and working conditions of the professionals of the a Mobile Emergency Service. Rev Lat Am-Enferm. 2011;19:1018-24. DOI: 10.1590/S0104-11692011000400022.

10. Bennett P, Williams Y, Page N, Hood K, Woollard M. Levels of mental health problems among UK emergency ambulance workers. Emerg Med J. 2004;21:235-6. DOI: 10.1136/emj.2003.005645.
11. Patterson PD, Suffoletto BP, Kupas DF, Weaver MD, Hostler D. Sleep quality and fatigue among prehospital providers. Prehosp Emerg Care. 2010;14(2):187-93. DOI: 10.3109/10903120903524971.

12. Santos Y, Porto F, Marques L, Tomaz A, Toledo R, Lucena N. Assessment of work ability of health professionals in the mobile emergency unit. Work. 2012;41:778-82. DOI: 10.3233/WOR-2012-0240-778.

13. Takeda E, Robazzi MLC. Occupational accidents among ambulance drivers in the emergency relief. Rev Lat Am Enfermagem. 2007; 15:439-445. DOI: 10.1590/S010411692007000300012 .

14. Alexandre NMC, Coluci MZO. Validade de conteúdo nos processos de construção e adaptaçãoo de instrumentos de medidas. Cien Saude Coletiva. 2011;16:3061-8. DOI: 10.1590/S1413-81232011000800006.

15. Thomas J, Nelson J. Métodos de pesquisa em atividade física. Porto Alegre: Artmed; 2002.

16. Sousa TF, Fonseca SA, José HPM, Nahas MV. Validade e reprodutibilidade do questionário Indicadores de Saúde e Qualidade de Vida de Acadêmicos (Isaq-A). Arq Cien Esporte. 2011;1(1):21-30. Disponível em: http://www. uftm.edu.br/revistaeletronica/index.php/aces/article/ view/254/361.

17. Schlademann SM, Meyer T, Raspe H. The test-retest reliability of a questionnaire on the occurrence and severity of back pain in a German population sample. Int J Public Health. 2008;53:96-103. DOI: 10.1007/s00038-008-6097-2.

18. Rubio DM, Berg-Weger M, Tebb SS, Lee ES, Rauch S. Objectifying content validity: conducting a content validity study in social work research. Soc Work Res. 2003;27(2). DOI: $10.1093 / \mathrm{swr} / 27.2 .94$.

19. Salmond SS. Evaluating the reliability and validity of measurement instruments. Orthop Nurs. 2008;27(1). DOI: 10.1097/01.NOR.0000310608.00743.54

20. Wynd CAS, Schmidt B, Schaefer MA. Two quantitative approaches for estimating content validity. West J Nurs Res. 2003;25(5):508-18. DOI: 10.1177/0193945903252998.

21. Hermida PMV, Araújo IEM. Elaboração e validação do instrumento de entrevista de enfermagem. Rev Bras Enferm. 2006;59:314-20. DOI: 10.1590/S003471672006000300012 . 
22. Alexandre NMC, Coluci MZO. Validade de conteúdo nos processos de construção e adaptação de instrumentos de medidas. Cien Saude Coletiva. 2011;16(7):3061-8. DOI: 10.1590/S1413-81232011000800006.

23. Aguiar OBD, Fonseca MJM, Valente JG. Confiabilidade (teste-reteste) da escala sueca do questionário demandacontrole entre trabalhadores de restaurantes industriais do estado do Rio de Janeiro. Rev Bras Epidemiol. 2010;13:21222. DOI: $10.1590 / \mathrm{S} 1415-790 \times 2010000200004$.
24. Dalmoro M, Vieira KM. Dilemas na construção de escalas tipo Likert: o numero de itens e a disposição infulenciam no resultado? RGO Rev Gestão Organ. 2013;6(3):161-74. Disponível em: http://bell.unochapeco.edu.br/revistas/ index.php/rgo/article/view/1386/1184.

25. Alexander DA, Klein S. Ambulance personnel and critical incidents. $\mathrm{Br} \mathrm{J}$ Psychiatry. 2001;178(1):76-81. DOI: 10.1192/bjp.178.1.76.

Recebido em: 14.07.15

Aceito em: 06.04.16 